\title{
Research on Architecture of Third Party Scientific and Technical Service Integration Platform under the Perspective of Supply Chain
}

\author{
Shuang Jiang \\ College of Management \\ Wuhan University of Science and Technology \\ Hubei, China \\ 1987403160@qq.com
}

\author{
Jing Wang \\ College of Management \\ Wuhan University of Science and Technology \\ Hubei, China \\ 297150678@qq.com
}

\begin{abstract}
In order to solve the existing problems of science and technology service industry and provide users with diversified, integrated and specialized science-tech services, from the perspective of supply chain, this article constructs a third party science-tech service integration platform dominated by third party science-tech service intermediary institution integrator, which consists of governments, universities and scientific research institutes, industry associations, industrial technology center, financial institutions and users. During the construction of the platform's logical and functional structure, function and service integration is achieved by the means of demand \& resource integration as well as portal \&process integration. Therefore, great innovation can be made to construct a new integrated platform by involving supply chain theory, and it's conducive to enrich the theoretical system of science-tech service.
\end{abstract}

Keywords-Supply chain; third party science-tech service intermediary institution integrator; integrated platform; platform architecture

\section{INTRODUCTION}

Science-tech service industry is becoming a new engine of China's economic growth. However, there exist some problems in science-tech service industry in China, for instance, the poor circulation of information, the dispersion of resources, the low conversion rate of scientific research achievements and difficulties among supply \& demand. Throughout the research of scholars as in $[1,2]$, it is found that the integration platform of science-tech services has become a new paradigm to eliminate the obstacles of information asymmetry and integrate the scientific and technological innovation resources as well as promote the transformation of scientific and technological achievements. Learn from the experience of domestic and foreign science and technology intermediary institutions[3,4], we found that the science and technology intermediary service agencies have a higher specialized and standardized management degree in aspects of the transformation of scientific and technological achievements, technology transfer \& diffusion and technology consulting, compared with the government and enterprises. Science and technology intermediary service agencies are of great significance to optimize the allocation of resources and to pave a way for supply\& demand docking of science and technology. With the transition of government function in our country, more and more scientific research project began to be managed by professional teams [5]. The technology integration and transformation platform, which was built by Beijing health industry and Incubation Center [6], connecting scientific research institutes and manufacturing enterprises and acting as a bridge between agriculture and technology, is a realistic model of science and technology intermediary organization integration platform.

With the development of global economic integration, the competition in twenty-first Century has been upgraded from the competition among enterprises to the competition between supply chain and supply chain. Nowadays supply chain integration platform has become a new model of effective integration of resources to gain competitive advantage. Yi Yatong Company as in [7] adopts one-stop supply chain management service mode to build globally integrated supply chain service platform, which has shown enormous market potential. Scholar Li Chilin [8] used the technology of Internet of things (IOT) to build an intelligent automobile supply chain logistics integrated platform, and it realized supply chain integrated and intelligent management in the automotive industry through information sharing among supply chain nodes. From their experiences, we've come to the conclusion that supply chain management theory is also applicable to the science-tech services industry. These concepts about "Demand as driving force" \&"using information supply chain business processes to achieve information sharing" and "play a gathering effect, to achieve win-win" meet requirements of China's science and technology services.

Therefore, from the perspective of supply chain, this paper intends to regard third party scientific and technical service intermediary institution integrator as leader to construct a third party scientific and technical service integration platform, cooperating with government, universities \&research institutes, industry associations, financial institutions along with industry technology center, based on the principle of win-win cooperation and market-oriented. 


\section{THIRD PARTY SCIENTIFIC AND TECHNICAL SERVICE INTEGRATION PLATFORM}

The third party scientific and technical service integration platform is a market demand-oriented integrated platform, which coordinates with government, universities \& research institutes, industry associations, financial institutions and industry technology center. Besides, it's an IT-based platform with the idea of resource integration, information sharing and professional service. The main purpose of the third party scientific and technical service integration platform is to provide some special services for users, which is various, integrated and specialized.

\section{THIRD PARTY SCIENTIFIC AND TECHNICAL SERVICE INTEGRATION PLATFORM COMPONENTS}

Third party scientific and technical service integration platform structure is as shown in Fig. 1 below.

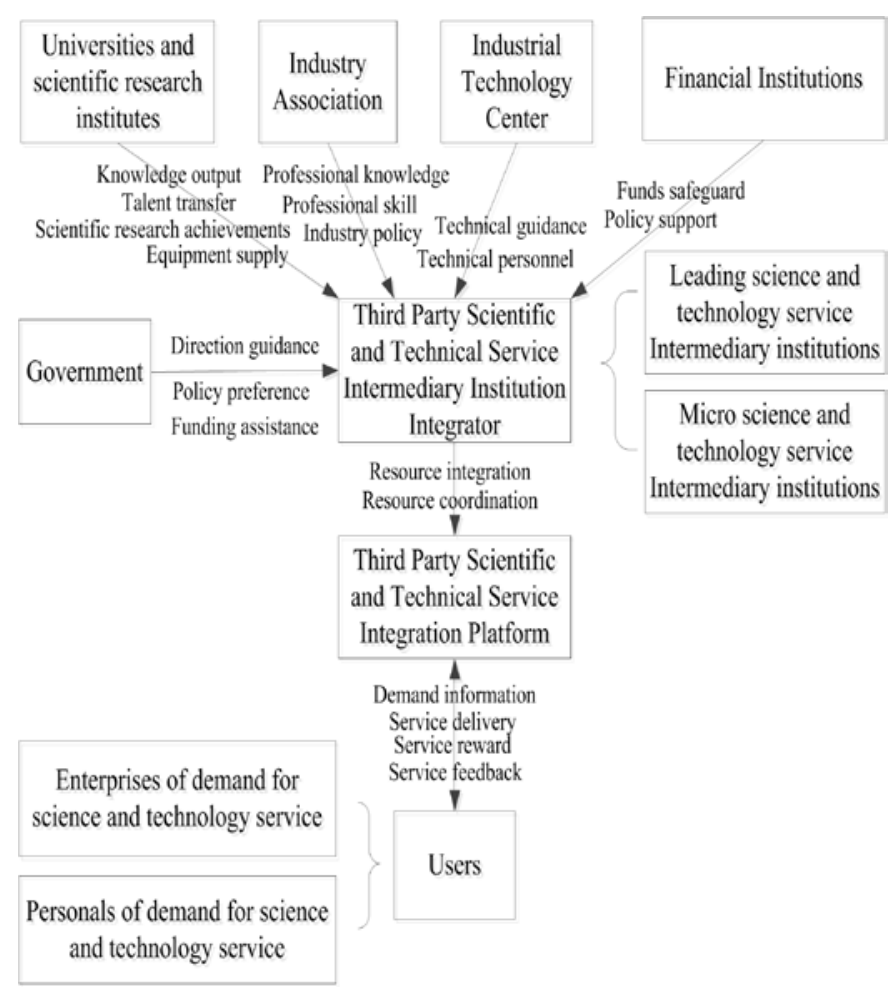

Fig. 1. Platform Components

\section{A. Third Party Scientific and Technical Service Intermediary Institution Integrator}

Third party scientific and technical service intermediary institution integrator is the core of third party scientific and technical service integration platform, which is constituted by large-scale and medium-sized third party technology service intermediaries. Its main responsibilities consist of five aspects. First, it collects the information of policy \& regulation, expert, scientific research and tech-facilities as well as users. Second, it constructs data Warehouse and import information into data warehouse with the help of IT technical staff. Third, it coordinates with other participants to strengthen the management of database information, platform application, customer's demand and business process. Fourth, based on user's needs, it invokes resources to provide users with online consultation service \& program design, service delivery\& feedback. Fifth, it expands offline users and makes visits to enterprise for excavating demand.

\section{B. Government}

Government is the core part of institutional innovation policy maker, and it guides other subjects' behavior. First, government should guide financial institutions to strengthen cooperation with third party scientific and technical service intermediary institution integrator. Second, it shall give stakeholder preferential policies support and financial assistance to attract more participants. Third, it could create a good atmosphere and strengthen the supervision of services quality.

\section{Universities and Scientific Research Institutes}

Universities \& research institutes are the birthplaces of knowledge \& scientific research achievements and talents. Teachers, students and retirees in universities\& research institutes are important parts of expert database. Writings and research papers have strong theoretical guidance, and scientific research patents can be transformed into actual productivity. Universities \&scientific research institutes are equipped with complete facilities, which is beneficial to conduct scientific and technological work.

\section{Industry Association}

Industry Associations are representative of entire sciencetech service industry. It could organize the various resources and coordinate its relationship with other subjects as well as participate in the formulation of some policies and regulations. Besides, it also provides professional skills and talents for third party scientific and technical service intermediary institution integrator to solve the various problems.

\section{E. Financial Institutions}

Financial institutions can provide strong financial support for platform's operating costs, staff salaries and incentives mechanism. In addition, it could give support for platform to broaden financing channels for small\& medium enterprises and provide risk management.

\section{F. Industrial Technology Center}

Industrial technology center has plentifultechnical personnel and high-level technology. Technical staffs can help to build the platform operation basis and establishing data warehouse. The platform operation basis included hardware configuration \& software installation, operating environment maintenance and security mechanisms \&QOS mechanisms. Data warehouse included policy \& regulations database, expert information database, scientific \& technological achievements information database, user information database, staff information database and difficulty collection database.

\section{G. Users}

Users are not only services requester but also information providers. They can be either individuals or enterprises. They pay for the services and publish feedback once they received the scientific and technical services. 


\section{THIRD PARTY SCIENTIFIC AND TECHNICAL SERVICE INTEGRATION PLATFORM ARCHITECTURE}

\section{A. Third Party Scientific and Technical Service Integration} Platform Logical Structure

Considering its integrated, shared and accessible features of the platform, this article uses Java 2 Platform Enterprise Edition architecture [9] and Web services architectures to design the logical architecture as follows in Fig.2.

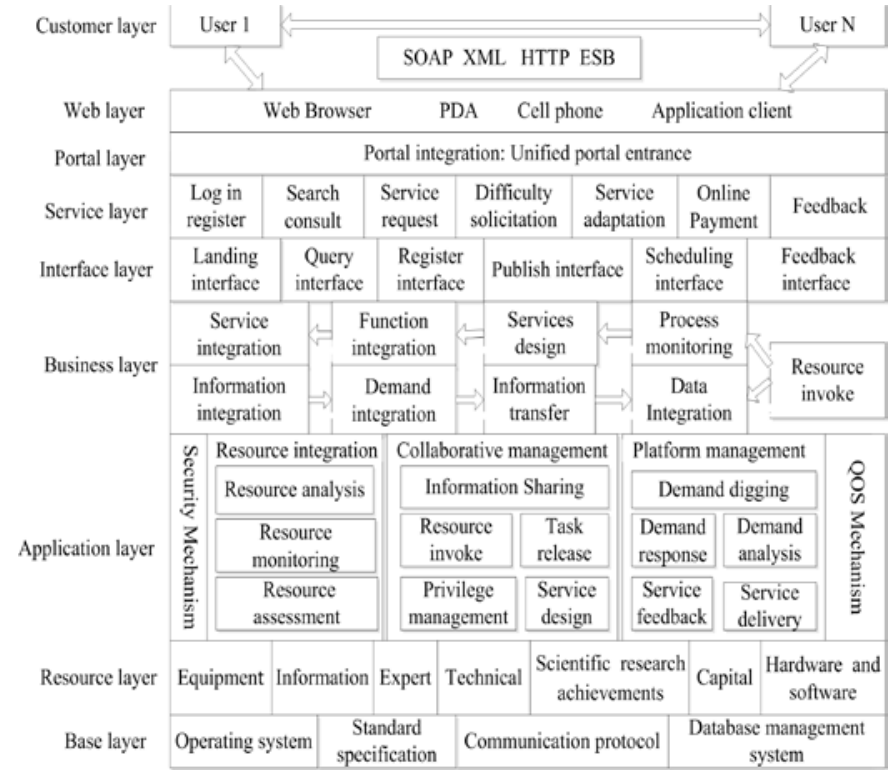

Fig. 2. Platform Logical Structure

The logical architecture of third party scientific and technical service integration platform contains nine levels: base layer, resource layer, application layer, business layer, interface layer, service layer, portal layer, Web layer and client layer. Base layer includes standard specification and communication protocol as well as operating \&database management system, which are the foundation and support of the platform. Resource layer includes various information databases, such as equipment, information, expert, technology, scientific achievements, capital, hardware and software, which provide resources for collaborative management and platform management of application layer. Application layer involves multiple application software and other legacy systems, which includes resource integration, collaborative management \& platform management and security system. In the security mechanism and QOS mechanism, third party scientific and technical service intermediary institution integrator firstly collate and analyze resources for resource integration; then it combine online way with offline way to dig out customer's demand; after that third party scientific and technical service intermediary institution integrator shall analyze and decompose these demand information to fulfill demand integration. Third party scientific and technical service intermediary institution integrator select suitable resources to design plan based on information integration. Before give user specific implementation plan, third party scientific and technical service intermediary institution integrator must make sure the program is reasonable and feasible. Finally it makes a monitoring \& tracking and pays attention to customer service feedback \& evaluation. Business layer refers to integrated business processes among the cross-border and cross-organizational heterogeneous subjects. After completing information integration and demand integration, the platform can use SOAP, XML, HTTP and other technologies to achieve the goal of data transmission among nodes in the supply chain, it also can utilize SOA to reassemble and invoke the resources in the resource layer. If user's demand match with the repository of the existing resources, third party scientific and technical service intermediary institution integrator directly invoke these resources; If not, third party scientific and technical service intermediary institution integrator is responsible for assigning tasks to each subject and strengthening the process of monitoring and tracking along with optimizing problems in the implementation of the program timely. Interface layer includes $\log$ in interface, query interface, register interface, publish interface, dispatch interface and feedback interface. User could $\log$ on the platform and query information through these interfaces. After registering as users can they post service request and publish service feedback \&evaluation, while platform staff can invoke resources through dispatch interface. In the service layer, customers can register, access to diverse information retrieval \& consultation, publish service requests, release difficulty solicitation, make online payment and publish feedback \&evaluation. Portal layer refers to the platform provide users with integrated portal through single sign on technology [10]. Users at client layer could enter the third party scientific and technical service integration platform through the web browser, PDA, application client.

\section{B. Third Party Scientific and Technical Service Integration Platform Functional Architecture}

According to the platform's logic architecture design the platform's function structure is as follows in Fig. 3.

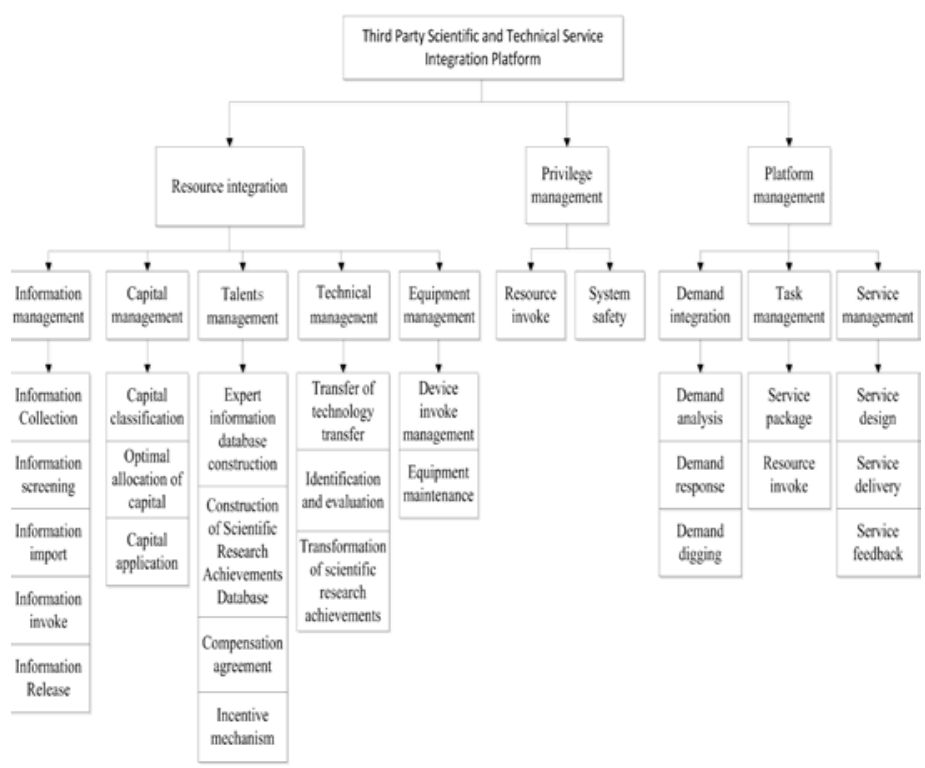

Fig. 3. Platform Function Structure 
Resources integration function is based on integrated management of information, technology, talents, capital, facilities to enhance resource reserves and ensure resource availability, which makes a guarantee for third party scientific and technical service integration platform to complete the needs of users. After third party scientific and technical service intermediary institution integrator finished the task of information collection, information filtering and information inputting, information could share in all-directions smoothly and fully. The capital not only provides a guarantee of funds for the platform's operation cost, salary reward and other expenditure but also opens up a new financing channel for small \& medium-sized enterprises, which come from government subsidies, financial support, Social fund-raising and service income. The establishment of compensation agreement and Incentive mechanism stimulate the work enthusiasm of staff, experts and technical staff. Equipment maintenance \& invocation management ensure the availability of equipment resources. Privilege management functions include resource invoke permissions and system security management rights. Only third party scientific and technical service intermediary institution integrator can invoke these integrated resources within the platform, in addition to information resources can be shared, other resources are limited. For example, universities can share various information resources on the platform but cannot utilize the funds privately. Only after registering and publishing service request can universities raise funds. Apart from third party scientific and technical service intermediary institution integrator, other participants have no right to arbitrarily tamper with or safeguard the platform's security system. For instance, technical center have right to give feedback to platform but no authority to maintain security vulnerabilities. Only third party scientific and technical service intermediary institution integrator is eligible to maintain system security. Platform management function includes demand integration and task \&service management. Demand integration contains user demand digging \& analysis and response. Task management involves service encapsulation and resource invocation. Service management includes service design, service delivery and service feedback. On the basis of demand integration, the task is decomposed and allocated; then resources are invoked for service design. After pledging service plan is feasible, third party scientific and technical service intermediary institution integrator hands over it to user through the platform and pays attention to user feedback.

\section{SUMMARY}

The third-party science-tech service integration platform provides a carrier for customers to convey their science-tech pursuit, which makes it easier to obtain customers' demand information. Besides, the platform can effectively optimize resource allocation among the supply chain nodes and promote information sharing in all directions, consequently reducing the waste of resource and promoting the quality of service. Furthermore, it can accelerate transformation of science-tech achievements and supply \& demand docking. This article made an innovation by involving supply chain theory and focusing the third party science-tech service Intermediary, which enriches the theoretical system of science and technology service. However, there are also some weaknesses; for example, there are lack of carefully introduction of the platform function and lack of detailed description of the platform operating mechanism as well as lack of in-depth explanation of platform integration required for a variety of information technology.

\section{REFERENCES}

[1] Liu Bo, Xia Xin, Chen Yandong, Research of Hospital Information Integration and Sharing Based on Integration Platform [J]. Hospital Digitization, 2013, 34 (7):46-48. (In Chinese)

[2] Zhang Xiaoliang, Jing Shenqi, Zhu Yongqian, The Practice of Hospital Data Integration Platform Construction[J].China Digital Medicine, (2016) . (In Chinese)

[3] Muhammad, Shajjad, Ahsana, Khaleel, Malikb, The Role of an Intermediary Agent in Technology Integration within Developing Countries: A Film Industry Perspective [J]. Procedia - Social and Behavioral Sciences, 2015, 195:151-156.

[4] Changli Li, Tony Lan, Shang-JyhLiu, Patent attorney as technology intermediary: A patent attorney facilitated model of technology transfer in developing countries [J]. World Patent Information 43, 2015,pp.62-73.

[5] http://www.gov.cn/zhengce/content/2015-01/12/content_9383.htm. (In Chinese)

[6] http://blog.sina.com.cn/618bl. (In Chinese)

[7] Zheng Yanling, Supply chain value added [M]. Beijing: Renmin University of China Press, 2013:213-222. (In Chinese)

[8] LI Chilin, LI Xiaoli, Research on the Intelligent Automobile Supply Chain Logistics Integration Platform based on the Internet of Things [D]. Wuhan University of Technology, 2012 (4). (In Chinese)

[9] Gonzalez Benjamin .A, Hike through a Post-EJB J2EE Web Application Architecture, Part III [J]. Computing in Science and Engineering, 2007, 9(1)pp.82-95.

[10] Hulme George V, BullSoft, Single Sign-On Technology Eases Data Access [J].Information week, 2000 (779):26. 